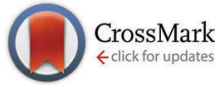

Cite this: J. Mater. Chem. B, 2017, 5, 1633

\title{
Tuning the local solvent composition at a drug carrier surface: the effect of dimethyl sulfoxide/ water mixture on the photofunctional properties of hypericin- $\beta$-lactoglobulin complexes
}

\author{
P. Delcanale, ${ }^{a}$ B. Rodríguez-Amigo, ${ }^{b}$ J. Juárez-Jiménez, ${ }^{c}$ F. J. Luque, ${ }^{c}$ \\ S. Abbruzzetti, ${ }^{\text {ad }}$ M. Agut, ${ }^{\text {b }}$ S. Nonell*b and C. Viappiani*ad
}

\begin{abstract}
Aggregation is a major problem for the anti-microbial photodynamic applications of hydrophobic photosensitizers since it strongly reduces the amount of singlet oxygen generated in aqueous solutions. Binding of hypericin (Hyp) to the milk whey protein $\beta$-lactoglobulin ( $\beta L G$ ), occurring at the two hydrophobic cavities located at the interface of the protein homodimer, can be exploited to confer water-solubility and biocompatibility to the photosensitizer. The introduction of a small amount of the organic cosolvent dimethyl sulfoxide (DMSO) leads to a remarkable improvement of the photophysical properties of the complex Hyp- $\beta$ LG by increasing its fluorescence emission and singlet oxygen photosensitization quantum yields. Surprisingly, the ability of the complex to photo-inactivate bacteria of the strain Staphylococcus aureus is strongly reduced in the presence of DMSO, despite the higher yield of photosensitization. The reasons for this apparently contradictory behavior are investigated, providing new insights into the use of carrier systems for hydrophobic photosensitizers.
\end{abstract}

Received 8th January 2017,
Accepted 20th January 2017

DOI: $10.1039 / \mathrm{c} 7 \mathrm{tb} 00081 \mathrm{~b}$

rsc.li/materials-b

\section{Introduction}

The use of photosensitization for antimicrobial purposes is gaining interest in medical applications for the treatment of localized infections, ${ }^{1,2}$ and for the decontamination and disinfection processes in the food industry. ${ }^{3}$

Photosensitization is a photo-reaction involving an otherwise non-toxic molecule, called a photosensitizer (PS), visible light and molecular oxygen. The absorption of a photon by the PS results in the formation of a triplet state (with yield $\Phi_{\mathrm{T}}$ ), showing a relatively long lifetime (usually in the $\mu$ s time scale). The triplet state is efficiently quenched by interaction with molecular oxygen leading to the production of cytotoxic reactive oxygen species (ROS), in most cases the non-radical electronicallyexcited singlet oxygen $\left({ }^{1} \mathrm{O}_{2}\right) .{ }^{4}$ The high reactivity of ${ }^{1} \mathrm{O}_{2}$ towards multiple cellular components (e.g. membrane lipids, proteins

\footnotetext{
${ }^{a}$ Dipartimento di Scienze Matematiche, Fisiche e Informatiche, Università di Parma, Parco Area delle Scienze 7A, 43124 Parma, Italy.

E-mail: cristiano.viappiani@unipr.it

${ }^{b}$ Institut Quimic de Sarrià, Universitat Ramon Llull, Via Augusta 390, 08017 Barcelona, Spain. E-mail: santi.nonell@iqs.url.edu

${ }^{c}$ Department of Nutrition, Food Science, and Gastronomy, and Institute of

Biomedicine, University of Barcelona, Av. Prat de la Riba 171,

08921 Santa Coloma de Gramenet, Spain

${ }^{d}$ NEST, Istituto Nanoscienze, Consiglio Nazionale delle Ricerche,

Piazza San Silvestro 12, 56127 Pisa, Italy
}

and nucleic acids) precludes the selection of photo-resistant species. ${ }^{5,6}$

Hypericin (Hyp) is a naturally occurring PS, found in the plants belonging to the genus Hypericum (e.g. St. John's wort). ${ }^{7}$ Hyp has proved to be efficient as an antibacterial, ${ }^{8-10}$ antiviral ${ }^{11}$ and antifungal ${ }^{19}$ photodynamic agent. When dissolved in organic solvents, Hyp is endowed with a good quantum yield of fluorescence emission $\left(\Phi_{\mathrm{F}}=0.35 \pm 0.02\right.$ in dimethyl sulfoxide $\left.(\mathrm{DMSO})^{12}\right)$ and singlet oxygen sensitization $\left(\Phi_{\Delta}=0.32\right.$ in ethanol, ${ }^{13} 0.39 \pm 0.01$ in methanol $\left.{ }^{14}\right)$ upon excitation with visible light. One of the main problems to overcome in the delivery of Hyp to targeted cells is the very low water-solubility that leads to the aggregation of the compound and almost complete loss of fluorescence emission and singlet oxygen sensitization. ${ }^{15,16} \mathrm{Hyp}$ is thus usually delivered by means of suitable carriers like liposomes ${ }^{17}$ or nanoparticles, ${ }^{10,18}$ in some cases endowed with targeting capabilities. ${ }^{19}$ Among other carriers, proteins are highly biocompatible systems that warrant bioavailability of the PS. We recently demonstrated that the complex between Hyp and apomyoglobin (apoMb) is an effective photosensitizing agent for Gram positive bacteria, ${ }^{20}$ with fluorescence properties that allow us to localize the PS with subdiffraction resolution on bacterial cells, using STED (Stimulated Emission Depletion) microscopy. ${ }^{21}$

We also demonstrated the use of $\beta$-lactoglobulin $(\beta \mathrm{LG})$ as a carrier for Hyp in aqueous solutions such as phosphate-buffered 
saline (PBS), with the advantage, relative to apoMb, that it could carry more Hyp molecules per protein unit. ${ }^{22}$ Since $\beta$ LG is one of the most abundant proteins of the bovine milk, its use is of special interest for antimicrobial applications in the dairy industry. However, although the complex Hyp- $\beta$ LG proved effective as a photosensitizing agent, its spectral properties and the low fluorescence and singlet oxygen quantum yields suggest that Hyp is not fully monomeric when bound to the protein, which prevents the full realization of its photosensitizing potential. ${ }^{22}$

Since $\beta$ LG can be coated with $\mathrm{DMSO}^{23}$ we hypothesize that the DMSO layer around $\beta \mathrm{LG}$ could provide a better environment for the bound Hyp molecules. In this work, we seek to study whether the use of DMSO as a cosolvent improves the photophysical properties of Hyp- $\beta \mathrm{LG}$, thereby assessing its potential as a photodisinfection agent for materials contaminated with bacteria. Indeed, the results confirm that DMSO as a cosolvent leads to an improvement in the fluorescence and singlet oxygen yields of bound Hyp. Surprisingly, the photo-inactivating action of the complex against bacteria was decreased. This apparently contradictory finding demonstrates that a high efficiency of ${ }^{1} \mathrm{O}_{2}$ photosensitization may not necessarily translate into higher photodynamic efficiency against bacteria.

\section{Results and discussion}

\section{Hyp and $\beta L G$ form a stable complex in a DMSO:PBS mixture}

$\beta$ LG spontaneously forms homodimers (2 $\beta \mathrm{LG})$ under physiological conditions and under the conditions encountered in milk processing. ${ }^{24,25}$ Dimerization involves the antiparallel alignment of the strand $\beta I$ (residues 163-167), not involved in the eight-stranded $\beta$-barrel, assisted by the formation of hydrogen bonds between the interacting strands, and the intersubunit interface is also shaped by the loop $\mathrm{AB}$, where residues Asp49 and Arg66 participate in salt bridges. As previously shown by molecular modelling, Hyp can a priori be accommodated in the two clefts formed at the interface of $2 \beta \mathrm{LG}$. In the narrower cleft, which is shaped by the $\alpha$-helix that precedes the strand $\beta \mathrm{I}$, Hyp can be inserted only as a monomer. The other cleft, formed by loops $\mathrm{AB}$ and $\mathrm{CD}$, which contains Trp77, is wider and might accommodate the binding of dimeric Hyp, and possibly higher order aggregates. ${ }^{22}$

Experimental proof for the formation of a stable complex between Hyp and 2 $\beta L G$ is provided by a combination of spectroscopic techniques. Hyp is readily soluble in polar organic solvents, like DMSO, where it shows a structured absorption spectrum, with two sharp bands at $555 \mathrm{~nm}$ and $599 \mathrm{~nm}$, and intense fluorescence emission with two bands centered at $604 \mathrm{~nm}$ and $653 \mathrm{~nm}$ (Fig. 1A and B, red). In PBS buffer, absorption bands are lowered in intensity and less structured, and the fluorescence emission is almost completely quenched (Fig. 1A and B, black), as a consequence of the aggregation of the molecule. In the presence of $2 \beta \mathrm{LG}$ in the PBS buffered solution, the structured absorption spectrum is partially recovered, with bands centered around $560 \mathrm{~nm}$ and $598 \mathrm{~nm}$, along with fluorescence emission (Fig. 1A and B, blue).

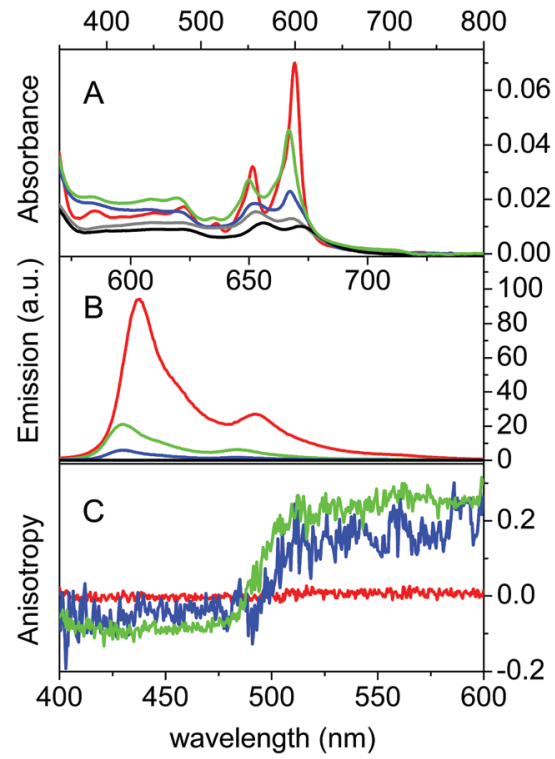

Fig. 1 Absorption (A), fluorescence emission (B) and fluorescence anisotropy (C) spectra of Hyp in DMSO (red), Hyp in PBS (black), Hyp in 20\% PBS-DMSO (grey), Hyp2 $\beta$ LG in PBS (blue) and Hyp2 $\beta L G$ in $20 \%$ PBSDMSO (green). The Hyp concentration is $4 \mu \mathrm{M}$ and that of $\beta L G$, when present, is $200 \mu \mathrm{M}$. Emission spectra are collected after excitation at $554 \mathrm{~nm}, T=20^{\circ} \mathrm{C}$. Fluorescence anisotropy spectra are obtained from excitation spectra $\left(\lambda_{\mathrm{em}}=620 \mathrm{~nm}, T=20^{\circ} \mathrm{C}\right)$.

As previously observed for apoMb $\mathrm{Mb}^{20}$ and bovine serum albumin, ${ }^{26}$ these facts are a consequence of the binding of Hyp to the protein, which can accommodate the molecule in a less-polar environment than water, thus preventing the aggregation. Time-resolved fluorescence emission measurements for Hyp in solution in the presence of $2 \beta \mathrm{LG}$ afford a biexponential decay with lifetimes $\left(\tau_{\mathrm{F}}\right)$ of $3.9 \mathrm{~ns}(35 \%)$ and $6.7 \mathrm{~ns}(65 \%)$, consistent with the values observed for Hyp bound to apoMb, where the high concentration of the longlived species was attributed to Hyp shielded from the aqueous solvent. ${ }^{20}$ As can be observed in Fig. 1C, Hyp in solution with $2 \beta \mathrm{LG}$ shows a non-zero anisotropy fluorescence excitation spectrum, indicating that the rotational diffusion of the molecule is limited because of the interaction with the protein scaffold. In comparison, Hyp dissolved in pure DMSO gives a zero anisotropy spectrum (Fig. 1C, red), due to the faster rotational diffusion.

Fluorescence Correlation Spectroscopy (FCS) provided additional proof for the formation of a stable complex between $2 \beta L G$ and Hyp. FCS is useful to determine the diffusion coefficient of molecules taking advantage of the spontaneous thermal fluctuation in the fluorescence emission intensity of the freely diffusing fluorophores in solution. ${ }^{27,28}$ Fig. $2 \mathrm{~A}$ shows the crosscorrelation function determined for $1 \mathrm{nM}$ Hyp in the presence of $30 \mu \mathrm{M} \beta \mathrm{LG}$. The cross-correlation signal is well described by a model comprising diffusional motion, on the $10 \mu \mathrm{s}-1 \mathrm{~ms}$ time scale, and triplet decay, occurring on the 1-10 $\mu$ s time scale. The diffusion coefficient of the dominant diffusing species is $D=70 \mu \mathrm{m}^{2} \mathrm{~s}^{-1}$, consistent with the value expected for Hyp bound to $2 \beta \mathrm{LG}$. 


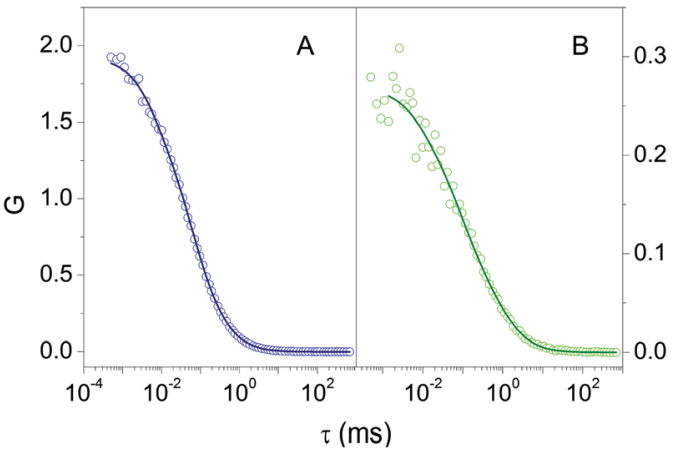

Fig. 2 Cross correlation functions for $1 \mathrm{nM}$ Hyp in PBS buffer in the presence of $30 \mu \mathrm{M} \beta \mathrm{LG}$ ( $\mathrm{A}$ - open circles) and $1 \mathrm{nM}$ Hyp in the mixture PBS-DMSO in the presence of $36 \mu \mathrm{M} \beta \mathrm{LG}$ (B - open circles). The solid lines are the results of the fit using a model comprising triplet state decay and diffusion. Excitation $475 \mathrm{~nm}$, detection at $670 \mathrm{~nm}$.

\section{Coating $\beta$ LG with DMSO improves the photofunctional properties of bound Hyp}

A key issue for potential applications in photosensitizationbased antimicrobial treatments is that the PS retains the ability to populate the triplet state upon photo-excitation and photosensitize the production of ${ }^{1} \mathrm{O}_{2}$. As shown in Table 1 , the complex Hyp2 $\beta L G$ in PBS buffer has a quantum yield for the triplet state formation $\Phi_{\mathrm{T}}=0.050 \pm 0.002$. The triplet state shows a lifetime decay $\left(\tau_{\mathrm{T}}\right)$ of around $10 \mu \mathrm{s}$, considerably longer than the value obtained for Hyp in DMSO $(1.4 \mu \mathrm{s})$ and similar to the one reported for Hyp bound to apoMb $(11.1 \mu \mathrm{s})$, where this relatively long lifetime was attributed to the shielding of Hyp from the solvent by the protein scaffold. ${ }^{20}$ Hyp $2 \beta$ LG photosensitizes ${ }^{1} \mathrm{O}_{2}$ with a yield $\Phi_{\Delta}=0.065 \pm 0.010$. The similar $\Phi_{\Delta}$ and $\Phi_{\mathrm{T}}$ values measured for Hyp2 $\beta \mathrm{LG}$ in PBS buffer indicate that the efficiency of the energy transfer between the triplet state of Hyp and ${ }^{3} \mathrm{O}_{2}$, leading to the production of ${ }^{1} \mathrm{O}_{2}$, is close to unity. However, the value retrieved for $\Phi_{\Delta}$ is quite low in comparison to the one observed for Hyp in pure DMSO $(0.28 \pm 0.05)$ or Hyp bound to apoMb $(0.14 \pm 0.03) .{ }^{31}$ According to molecular modeling studies, the reduced fraction of active PS may be due to the preferential binding of Hyp, possibly as an aggregate, to the wide cleft in $2 \beta \mathrm{LG}$, where it is stacked against Trp77. Moreover, when bound to the wide cleft, one face of Hyp is exposed to the aqueous solvent, an interaction which may be detrimental to the photophysics of the compound.

To overcome these limitations we have taken advantage of the preferential interactions between DMSO and 2 $3 \mathrm{LG}$. Since DMSO (up to $50 \%$ ) is known to coat $2 \beta \mathrm{LG}$ without affecting its structure to any appreciable extent, ${ }^{23}$ we have introduced a small amount of DMSO as a cosolvent, with the aim of providing a better environment to bound Hyp, thus increasing the fraction of photo-active bound molecules. We chose a DMSO concentration of $20 \%\left(V_{\mathrm{DMSO}} / V_{\mathrm{PBS}}\right)$, corresponding to $\sim 17 \%$ ( $\left.V_{\mathrm{DMSO}} / V_{\text {tot }}\right)$, well below the limit at which denaturation is observed. Molecular dynamics (MD) simulations performed for dimeric $\beta \mathrm{LG}$ in a DMSO/water mixture at this concentration reveal that DMSO molecules tend to fill specific pockets in hydrophobic patches of the protein surface, including the areas corresponding to the narrow and wide clefts formed at the interface of the interacting monomers (Fig. 3). This agrees with the experimental finding that at low DMSO concentrations there is a negative preferential binding of DMSO molecules, ${ }^{23}$ reflecting the favorable interactions of DMSO with aliphatic and aromatic residues. Furthermore, mapping of the highly populated binding sites for organic probes is valuable to identify 'hot spots' for the binding of small molecules. ${ }^{30-33}$ The distribution of DMSO molecules on the protein surface is consistent with the observation, based on $\mathrm{THz}$ spectroscopy, that $\beta$ LG is a relatively hydrophobic protein not displaying an extended long-range hydration dynamics, ${ }^{34}$ as is observed for other hydrophilic proteins. ${ }^{35,36}$

Hence, these results suggest that DMSO might stabilize the attachment of monomeric Hyp to the clefts located at the interface of $2 \beta \mathrm{LG}$.

The formation of a stable Hyp $2 \beta L G$ complex in the binary DMSO-PBS mixture was confirmed by fluorescence anisotropy and FCS measurements. The anisotropy spectrum (Fig. 1C, green) shows non-zero values, consistent with the ones observed for Hyp2 $\beta L G$ in pure PBS buffer. The cross-correlation function measured using FCS for Hyp2 $\beta \mathrm{LG}$ (10 nM Hyp with $36 \mu \mathrm{M} \beta \mathrm{LG}$ )

Table 1 Photophysical parameters of Hyp and Hyp2 $\beta$ LG in PBS or 20\% PBS-DMSO incubated with S. aureus cells

\begin{tabular}{|c|c|c|c|c|c|c|c|}
\hline Sample & Solvent & $\tau_{\mathrm{F}}(\mathrm{ns})$ & $\Phi_{\mathrm{F}}$ & $\tau_{\mathrm{T}}(\mu \mathrm{s})$ & $\Phi_{\mathrm{T}}$ & $\tau_{\Delta}(\mu \mathrm{s})$ & $\Phi_{\Delta}$ \\
\hline Нyp & DMSO & $5.5 \pm 0.1(100 \%)$ & $0.35 \pm 0.02^{12}$ & $1.4 \pm 0.1^{a}$ & $0.35^{13}$ & $5.5 \pm 0.1$ & $0.28 \pm 0.05^{29}$ \\
\hline Нур & $\mathrm{PBS}+S$. aureus & $\begin{array}{l}0.2(48 \%) \\
4.2(52 \%)\end{array}$ & & & & & \\
\hline Нyр & $\begin{array}{l}\text { PBS-DMSO } 20 \% \\
\text { +S. aureus }\end{array}$ & $\begin{array}{l}3.5(100 \%) \\
0.5(48 \%) \\
3.6(52 \%)\end{array}$ & & & & & \\
\hline Hyp $2 \beta L G$ & PBS & $\begin{array}{l}3.9(35 \%) \\
6.7(65 \%)\end{array}$ & $0.03 \pm 0.01$ & $\begin{array}{l}10 \pm 2^{a} \\
8.6^{b} \\
9.6^{c}\end{array}$ & $0.050 \pm 0.002$ & $2.3 \pm 0.1$ & $0.065 \pm 0.010$ \\
\hline & $+S$. aureus & $\begin{array}{l}4.6(45 \%) \\
7.0(55 \%)\end{array}$ & & $8 \pm 1^{a}$ & & & \\
\hline Hyp $2 \beta L G$ & $\begin{array}{l}\text { PBS-DMSO } 20 \% \\
+ \text { S. aureus }\end{array}$ & $\begin{array}{l}0.2(20 \%) \\
5.6(80 \%) \\
5.5(100 \%)\end{array}$ & $0.06 \pm 0.01$ & $\begin{array}{l}8.2 \pm 0.4^{a} \\
8.4^{b} \\
7.5^{c} \\
9 \pm 1^{a}\end{array}$ & $0.170 \pm 0.002$ & $2.5 \pm 0.1$ & $0.12 \pm 0.05$ \\
\hline
\end{tabular}

${ }^{a}$ Laser flash Photolysis. ${ }^{b}$ Time-resolved NIR phosphorescence detection. ${ }^{c}$ Fluorescence correlation spectroscopy. 


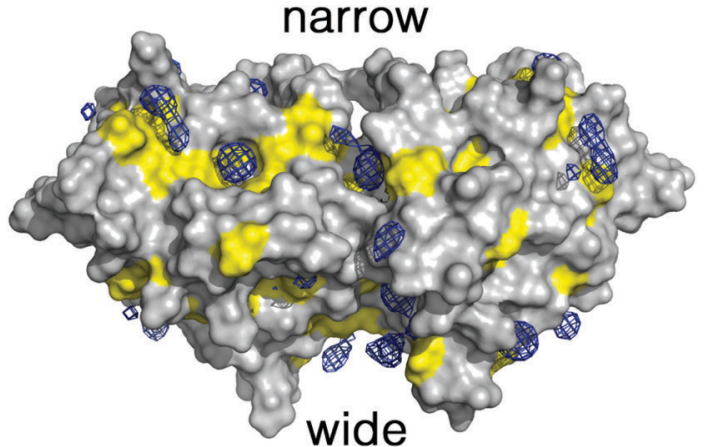

Fig. 3 Preferential distribution of DMSO molecules around the protein surface of dimeric $\beta \mathrm{LG}$. Blue isocontours denote a DMSO density of $0.3 \mathrm{~g} \mathrm{~cm}^{-3}$. Yellow areas denote the surface contribution arising from amino acids with hydrophobic side chains. The location of the narrow and wide clefts at the protein-protein interface is also shown.

in the mixture PBS-DMSO (Fig. 2B), is well-described by a model considering molecular diffusion and triplet decay. The diffusion coefficient of the dominant species is $D=40 \mu \mathrm{m}^{2} \mathrm{~s}^{-1}$. The lower value of $D$ observed in PBS-DMSO with respect to the pure PBS buffer arises from the higher viscosity of the mixture and the different hydrodynamic radius of the DMSO-coated protein..$^{37,38}$ The spectroscopic properties of the complex Hyp2 $\beta$ LG considerably change in the DMSO:PBS mixture: the absorption spectrum (Fig. 1A, green) is more structured than that in pure PBS, with absorption bands similar to those observed for Hyp dissolved in pure DMSO, and the intensity of the fluorescence emission is increased (Fig. 1B, green). As can be observed in Table 1, the presence of DMSO induces a nearly two-fold increase in the values of $\Phi_{\mathrm{F}}, \Phi_{\mathrm{T}}$, and $\Phi_{\Delta}$, which become comparable to those observed when Hyp is bound to apoMb. ${ }^{21}$ Table 1 also shows that the fluorescence decay of Hyp2 $\beta$ LG in DMSO:PBS occurs with a biexponential relaxation, where the main component has a 5.6 ns lifetime, identical to the one of Hyp in DMSO. This is in keeping with the picture derived from MD simulations, where Hyp bound to $2 \beta \mathrm{LG}$ is coated by DMSO. The minor fast component (lifetime $0.3 \mathrm{~ns}$ ) may arise from scattering.

The effect of local solvent composition on protein bound Hyp has been discussed in the case of human and bovine serum albumins, where the network of hydrogen bonds has been suggested to be responsible for the observed solvatochromism. ${ }^{42}$ While the presence of DMSO in close vicinity of the bound Hyp may affect the excited state proton transfer of the compound, and thus modulate its photophysics, ${ }^{39-41}$ the above results suggest that disruption of Hyp aggregates by specific DMSO solvation may be even more relevant.

Given the similar concentration of dissolved molecular oxygen in PBS buffer and in the 20\% DMSO-PBS mixture, ${ }^{43}$ the Hyp2 $\beta$ LG complex can be considered more efficient in the photosensitization of ${ }^{1} \mathrm{O}_{2}$ when assembled in a PBS-DMSO mixture.

The increment observed in the values of $\Phi_{\mathrm{F}}, \Phi_{\mathrm{T}}$ and $\Phi_{\Delta}$ for Hyp2 $\beta L G$ in DMSO-PBS cannot be attributed to a larger fraction of monomeric free Hyp molecules in the mixture, since the spectra recorded for Hyp alone (Fig. 1; grey curves) show the
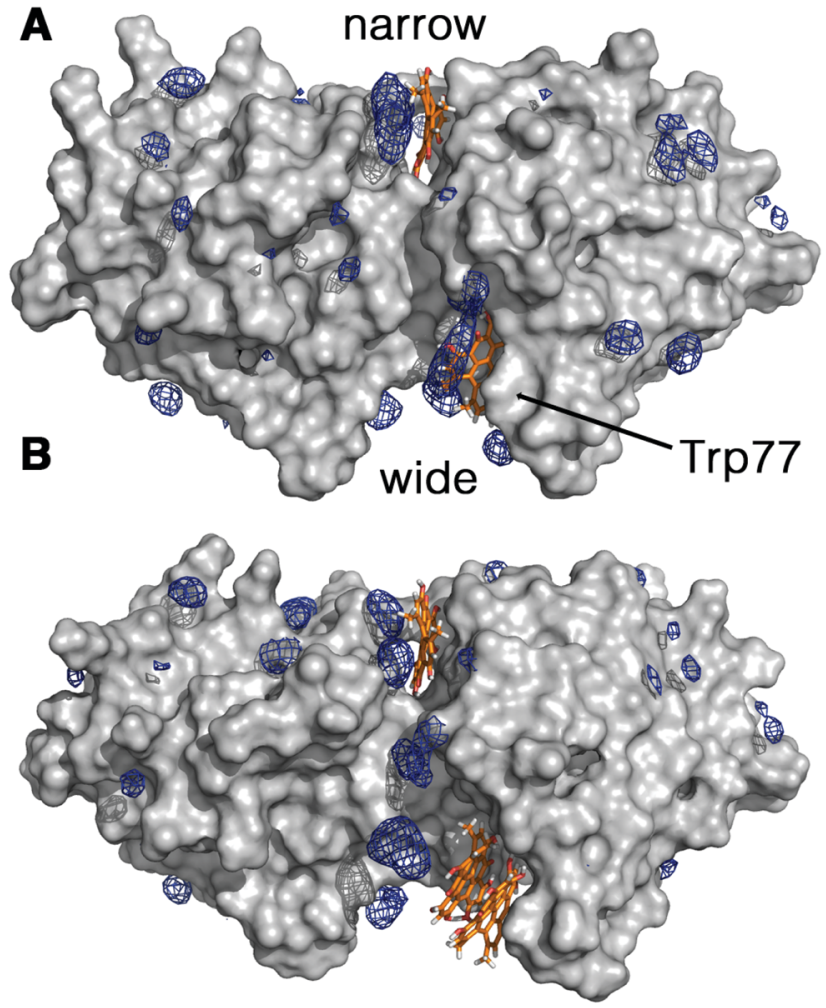

Fig. 4 Preferential distribution of DMSO molecules around the protein surface of dimeric $\beta L G$ bound to Hyp molecules as monomers in the narrow cleft, and either (A) monomers or (B) dimers in the wide cleft. Blue isocontours denote a DMSO density of $0.3 \mathrm{~g} \mathrm{~cm}^{-3}$. Hyp molecules are shown as orange-based sticks.

typical broadened and weaker absorption bands, a barely detectable fluorescence emission $\left(\Phi_{\mathrm{F}}<0.001\right)$, and a negligible formation of triplet state. Thus, the increased quantum yields observed in the presence of DMSO indicate a larger fraction of better solvated Hyp molecules bound to the protein. In fact, molecular modelling studies support the structural integrity of the Hyp2 $\beta \mathrm{LG}$ complex. As noted in Fig. 4, which shows the last snapshot collected at the end of a MD (250 ns) simulation, the presence of DMSO neither alters the binding of monomeric Hyp to the narrow cleft nor impedes the binding of Hyp, either as a monomer or a dimer, to the wide cleft, where it is stacked against the indole ring of Trp77 (the average distance between the Hyp and indole rings is close to $4.8 \AA$ A). Although the present results do not allow us to discern the relative stability of the binding to the two clefts, it is worth noting the large population of DMSO molecules around bound Hyp, especially for the monomeric species in the narrow and wide clefts (blue contour in Fig. 4). Moreover, monomeric Hyp is deeply inserted into the wide cleft, while the larger size of dimeric Hyp leads to a more superficial interaction with the protein. The preferential solvation by DMSO molecules is expected to result in a more effective shielding of bound Hyp from surrounding water molecules, which would stabilize the binding of monomeric Hyp to the protein. 


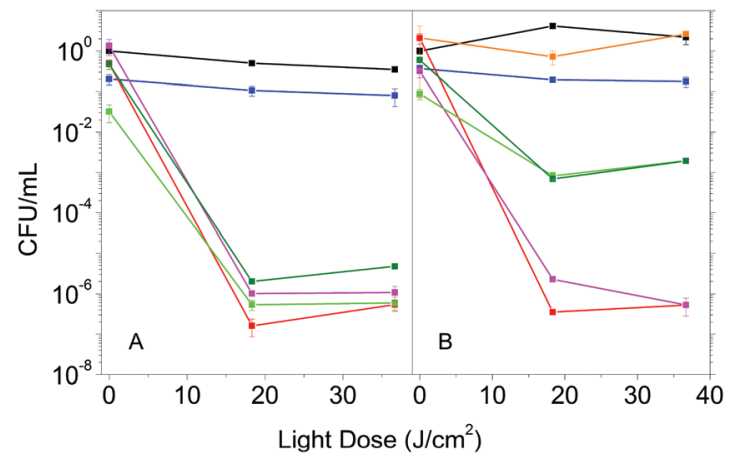

Fig. 5 Photoinactivation results for $S$. aureus cells in suspension incubated with: $4 \mu \mathrm{M}$ Hyp (red), $4 \mu \mathrm{M}$ Hyp and washed by centrifugation (magenta), Hyp $4 \mu \mathrm{M}$ complexed with $\beta L G 200 \mu \mathrm{M}$ (green), Hyp $4 \mu \mathrm{M}$ complexed with $\beta L G 200 \mu \mathrm{M}$ and washed by centrifugation (dark green). The same experiments are performed in a PBS buffered solution (A) and in a 20\% PBS-DMSO mixture (B). Controls are made for $S$. aureus suspension in PBS (black), in 20\% PBS-DMSO (orange), and incubated with $\beta L G$ $200 \mu \mathrm{M}$ (blue).

\section{Antimicrobial activity of the complex between $\beta$ LG and Hyp}

The enhanced photophysical properties in the presence of DMSO suggest that Hyp2 $\beta$ LG may have improved antimicrobial activity. We then compared the photoinactivation of $S$. aureus suspensions incubated with Hyp in DMSO, Hyp2 3 LG in PBS and Hyp2 $\beta \mathrm{LG}$ in the mixed solvent PBS-DMSO. In addition, after the incubation period, suspensions were centrifuged and the pellet containing the cells was re-suspended in an equal volume of solvent, while the supernatant was removed by suction. This washing procedure allows for the removal of the species that are not tightly combined to the cells after the incubation, and thus can be separated by centrifugation.

In pure PBS buffer, Hyp2 $\beta L G$ induces a relevant inactivation of $S$. aureus, with a decrease of $\sim 6 \log$ units in the number of bacterial CFU upon irradiation (Fig. 5A, green). This effect is comparable to the one observed when free Hyp in pure DMSO is added to the bacteria suspension (Fig. 5A, red), whose antimicrobial activity against Gram-positive bacteria is known. ${ }^{8,9,44,45}$ The results obtained for the washed suspensions are very similar: a decrease of $\sim 6 \log$ units in the number of CFU for free Hyp (Fig. 5A, magenta) and 5 to $6 \log$ units for Hyp2 $\beta \mathrm{LG}$, with a slight reduction of the dark toxicity (Fig. 5A, dark green). Fig. 5B shows the results of the experiments carried out using the $20 \%$ PBSDMSO mixture as a solvent. Control experiments demonstrated that the presence of DMSO at this concentration is not toxic for these bacteria (Fig. 5B, orange). As observed in pure PBS buffer, when free Hyp in pure DMSO is added, excellent photoinactivation is obtained, with a reduction of 6-7 log units in the number of CFU (Fig. 5B, red). No substantial change occurs after washing by centrifugation (Fig. 5B, magenta). Surprisingly, the complex Hyp2BLG considerably reduces its photodynamic action against $S$. aureus in the mixture PBS-DMSO, inducing a decrease in the number of CFU of $\sim 3 \log$ units upon irradiation (Fig. 5B, green). This result is in apparent contradiction with the measured value of $\Phi_{\Delta}$ of the complex, which is about two-fold higher in the mixed solvent PBS-DMSO than in pure PBS.
The different photodynamic activity towards $S$. aureus in the mixed solvent PBS-DMSO does not arise from a reduced photostability of the complex in the mixed solvent as compared to PBS. Exposing Hyp2 $\beta$ LG to the same light dose as used in the photoinactivation experiments leads to the same level of bleaching of the compound in PBS and in PBS-DMSO (data not shown).

\section{Spectroscopic analysis in the presence of bacteria}

The comparison of the fluorescence emission spectra in Fig. 6 can provide some indications about the distribution of Hyp in the presence of $S$. aureus cells. As previously discussed, free Hyp is largely aggregated in PBS buffer and no fluorescence can be detected at the concentration used (Fig. 6A, red). When free Hyp is placed in the mixture PBS-DMSO, the fluorescence emission is detectable with a maximum at $593 \mathrm{~nm}$, but still weak (Fig. 6B, red). After incubation with bacterial cells, the emission spectra of free Hyp undergo minor but important changes: a slight increase in the emission is observed in pure PBS (Fig. 6A, black, peak at $598 \mathrm{~nm}$ ), while an increase of the emission and a band-shift are observed in the mixture PBS-DMSO (Fig. 6B, black, peak at $598 \mathrm{~nm}$ ). Fluorescence decay is very similar for bacteria bound Hyp, either with or without DMSO (Table 1). This indicates re-distribution of Hyp, where Hyp molecules are placed in a different environment than the bulk solvent, provided by the cell.

The interaction between Hyp or Hyp2 $\beta \mathrm{LG}$ and $S$. aureus cells is crucial for the photodynamic inactivation induced by the photosensitizing species.

As previously discussed, the complex Hyp $2 \beta L G$ in pure PBS is considerably more fluorescent than free Hyp (Fig. 6A, green), and its emission becomes even more intense in PBS-DMSO (Fig. 6B, green). No significant changes in the emission spectra of Hyp2 $\beta$ LG are detected after incubation with $S$. aureus cells, either in pure PBS (Fig. 6A, cyan) or PBS-DMSO (Fig. 6B, cyan). When solutions are washed by means of centrifugation after incubation with bacteria, the fluorescence emission is largely reduced (Fig. 6A and $\mathrm{B}$, blue), becoming comparable with the

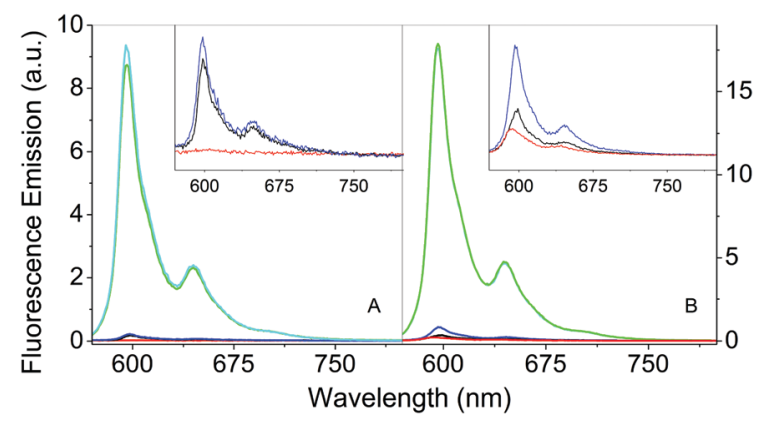

Fig. 6 Fluorescence emission spectra in PBS buffer (A) and in the 20\% PBS-DMSO mixture (B) for Hyp $4 \mu \mathrm{M}$ (red), Hyp $4 \mu \mathrm{M}$ incubated with S. aureus (black), Hyp $4 \mu$ M complexed with $\beta L G 200 \mu M$ (green), Hyp $4 \mu M$ complexed with $\beta L G 200 \mu \mathrm{M}$ incubated with S. aureus (cyan), Hyp $4 \mu \mathrm{M}$ complexed with $\beta L G 200 \mu \mathrm{M}$, incubated with S. aureus and washed by centrifugation (blue). The insets represent a zoom of the less intense signals. Excitation at $554 \mathrm{~nm}$. 
one observed for free Hyp incubated with bacteria. The small but clearly detectable emission spectra of the washed bacteria indicate that an appreciable fraction of Hyp2 $\beta L G$ is tightly bound to the bacteria. The position of the emission peak for this fraction is very similar in the absence $(598 \mathrm{~nm})$ and in the presence of DMSO $(597 \mathrm{~nm})$. All of the above results are unchanged whether bacteria are incubated with Hyp2 $\beta L G$ at room temperature, or at $37^{\circ} \mathrm{C}$.

When $S$. aureus cells are incubated with Hyp2 $\beta$ LG in PBS, time resolved fluorescence emission from the PS occurs through a biexponential relaxation with similar weights as, and slightly longer lifetimes than, those observed in the absence of bacteria (Table 1). This fact indicates that the fluorophore is kept in the same type of environment with or without bacteria, provided by the protein scaffold. Similarly, the fluorescence decay observed for $S$. aureus incubated with Hyp2 $\beta$ LG in PBS-DMSO is essentially the same as that observed for Hyp2 $\beta$ LG in PBS-DMSO and for Hyp in DMSO (Table 1). This suggests that the patches of DMSO, coating the hydrophobic areas of the protein surface, persist also when Hyp2 $\beta L G$ is bound to the bacteria.

Importantly, triplet decay of Hyp2 $\beta$ LG bound to $S$. aureus is very similar in PBS and PBS-DMSO, with a lifetime of approx. $9 \mu \mathrm{s}$, identical to the value measured in the absence of bacteria (Table 1). The long lifetime of the triplet state indicates that Hyp remains bound to the protein carrier, since transfer to the bacterial membrane would lead to a shorter triplet state lifetime (approx. 1-2 $\mu \mathrm{s}$ ).

Finally, the interaction between Hyp2 $3 \mathrm{LG}$ and $S$. aureus cells is further confirmed by FCS experiments, which demonstrate for all these samples the presence of fluorescent species with a diffusion coefficient consistent with that of large objects such as $S$. aureus cells (data not shown).

Since experimental evidence suggests that the complex between Hyp and $2 \beta L G$ remains intact even after binding to $S$. aureus, the different type of interaction of the whole complex Hyp2 $\beta$ LG coated by DMSO with the cells is most likely the reason for the reduced efficiency in the microbial photoinactivation. In spite of the higher amount of photosensitized ${ }^{1} \mathrm{O}_{2}$ by Hyp2 $\beta \mathrm{LG}$, the DMSO coating thus appears to decrease the bioavailability of the photoactive compound for yet to be understood reasons.

\section{Conclusions}

The complex between the dimeric milk whey protein $\beta$ LG and the natural photosensitizer Hyp has been fruitfully used as photoactive system in antibacterial photosensitization-based treatments. ${ }^{22}$ However, the presence of the wide cleft, capable of accommodating more than one PS molecule, and the partial exposure of bound Hyp to the aqueous environment lowers both triplet and fluorescence quantum yields, in comparison with cases where bound Hyp is strictly monomeric and the shielding from solvent is more efficient. ${ }^{20}$

Taking advantage of the known peculiar solvation properties of $\beta \mathrm{LG}$, we show that it is possible to coat the dimeric protein with the cosolvent DMSO, which provides a better environment for the bound Hyp molecules. While this increases fluorescence, triplet, and singlet oxygen yields, it turns out that the presence of DMSO is detrimental to the antibacterial treatment. This most likely arises from a change in the interaction between Hyp2 $\beta$ LG and the cellular constituents, which prevents the DMSO coated nanostructure from reaching more photosensitive regions in the bacterial wall.

The present work demonstrates that the improved photophysical properties of the delivery system are not necessarily translated into more effective photoinactivation of bacterial cells. The overall efficiency is the result of a combination of suitable photosensitization yield and interaction with the target environment.

\section{Experimental section}

Hypericin was obtained from HWI Analytik GmbH (Ruelzheim, Germany). $\beta$-Lactoglobulin (isoform B) from bovine milk was obtained from Sigma Aldrich (St. Louis, MO). All samples were used as received.

For the spectroscopic studies on the complex Hyp2 $3 \mathrm{LG}$, we choose experimental conditions comprising an excess of protein, so that the fraction of free Hyp molecules is negligible. Typical experimental values are $[\beta \mathrm{LG}]=200 \mu \mathrm{M}$, corresponding to $[2 \beta \mathrm{LG}] \approx 86 \mu \mathrm{M}$, and $[\mathrm{Hyp}]=4 \mu \mathrm{M}$. Under these conditions, $\sim 86 \%$ of $\beta \mathrm{LG}$ is found as a homodimer (dimerization constant $k_{\mathrm{D}}=8.6 \mu \mathrm{M}^{24}$ ) and $\sim 97 \%$ of Hyp is bound to the protein (dissociation constant $k_{\mathrm{d}}=0.71 \pm 0.03 \mu \mathrm{M}^{22}$ ). For FCS experiments, Hyp was kept at $1 \mathrm{nM}$ in the presence of $30 \mu \mathrm{M} \beta \mathrm{LG}$.

\section{General spectroscopic instrumentation}

Absorption spectra were recorded using a Jasco V-650 (Jasco Europe). Fluorescence spectra were recorded using a SpexFluoromax 4 (Horiba Jobin Yvon, Edison, NJ) or a Perkin Elmer LS50 spectrofluorometer (PerkinElmer, Waltham, MA). For steady state anisotropy studies, two Glan Taylor polarizers were placed before sample excitation and before fluorescence detection. Fluorescence decays were recorded by using a Fluotime 200 time-correlated single photon counting system (PicoQuant GmbH, Berlin, Germany) with pulsed LED excitation at 457 or $375 \mathrm{~nm}$ and detection via a PicoQuant PMA 182-M single photon detector.

\section{Singlet oxygen measurements}

The time-resolved ${ }^{1} \mathrm{O}_{2}$ phosphorescence signals and $\Phi_{\Delta}$ values were determined by direct detection of ${ }^{1} \mathrm{O}_{2}$ phosphorescence at 1275 nm using a modified PicoQuant Fluotime 200 system. A diode-pumped pulsed Nd:YAG laser (FTSS355-Q, Crystal Laser, Berlin, Germany) working at a $10 \mathrm{kHz}$ repetition rate $\left(\lambda_{\mathrm{exc}}=\right.$ $532 \mathrm{~nm}, 1.2 \mu \mathrm{J}$ per pulse) was used for excitation; the NIR luminescence exiting from the sample was detected at $90^{\circ}$ by using a H9170-45 NIR-PMT module (Hamamatsu) working in photon counting mode and a NanoHarp 250 multichannel scaler (PicoQuant, Germany). The time-resolved phosphorescence 
signals, showing the typical rise and decay, were fitted using the equation:

$$
S=S_{0} \frac{\tau_{\Delta}}{\tau_{\Delta}-\tau_{\mathrm{T}}}\left(\mathrm{e}^{-t / \tau_{\Delta}}-\mathrm{e}^{-t / \tau_{\mathrm{T}}}\right)+y_{0}
$$

where $\tau_{\mathrm{T}}$ and $\tau_{\Delta}$ are the lifetime of the photosensitizer triplet state and of ${ }^{1} \mathrm{O}_{2}$, respectively, $y_{0}$ is an offset due to instrument dark counts and $S_{0}$ is an instrumental quantity proportional to $\Phi_{\Delta}$. For $\Phi_{\Delta}$ measurements, Rose bengal (RB) and 5,10,15,20tetrakis(1-methyl-4-pyridinium)porphinetetra( $p$-toluenesulfonate) (TMPyP) were used as references. The sample and references were dissolved in the same solvent and air-equilibrated. Reference concentrations were adjusted so that the value of absorbance $(<0.1)$ at the excitation wavelength resulted to be the same as that of the sample. Time-resolved signals were acquired and fitted using eqn (1). The value for $\Phi_{\Delta}$ was then retrieved comparing the $S_{0}$ values for the sample and references: ${ }^{46}$

$$
\Phi_{\Delta, \text { sample }}=\Phi_{\Delta, \text { ref }} \frac{s_{0, \text { sample }}}{s_{0, \text { ref }}}
$$

\section{Fluorescence correlation spectroscopy (FCS)}

FCS experiments were performed using a Microtime 200 system from PicoQuant, based on an inverted confocal microscope (Olympus IX70) and equipped with two SPADs (single photon avalanche diodes) used in the cross-correlation mode. Excitation was achieved by using a $475 \mathrm{~nm}$ picosecond diode laser. Fluorescence emission by Hyp was collected through a bandpass filter and split with a 50/50 splitter between the two detection channels. The Hyp concentration was kept in the nM range, so that only a few molecules (always below 10) were detected in the confocal volume.

\section{Laser flash photolysis}

Triplet state decay of Hyp was monitored at $520 \mathrm{~nm}$ after photoexcitation with the second harmonic $(532 \mathrm{~nm})$ of a nanosecond Nd:YAG laser (Spectron Laser) using a previously described setup. ${ }^{47}$

\section{Molecular modeling}

Set up of the DMSO/water ( $17 \% \mathrm{v} / \mathrm{v})$ solvent box. Following the procedure reported by Alvarez and Barril ${ }^{48}$ a single molecule of DMSO was built using the Molecular Operating Environment (MOE) ${ }^{49}$ and optimized at the $\mathrm{HF} / 6-31 \mathrm{G}(\mathrm{d})$ level of theory using Gaussian $09 .^{50}$ Partial charges were derived for the optimized structure using the RESP procedure ${ }^{51,52}$ and atom types were assigned according to the general amber force field ${ }^{53}$ using Antechamber. ${ }^{54}$ The initial DMSO/water $(17 \% \mathrm{v} / \mathrm{v})$ box was obtained by solvating 6 DMSO molecules in a box of 88 TIP3P ${ }^{55,56}$ water molecules, minimized (3500 steps of steepest descent followed by 6400 steps of a conjugate gradient) and thermalized in three steps of 100 ps where the temperature was gradually increased from $50 \mathrm{~K}$ to $298 \mathrm{~K}$ in the NVT ensemble. Next, the volume of the box was equilibrated under constant pressure and temperature in two stages of $100 \mathrm{ps}$. Throughout all pre-equilibration stages, the temperature was controlled by using a Langevin thermostat with a collision frequency of $3 \mathrm{ps}^{-1}$, long-range electrostatics were treated using particle mesh Ewald summation, a cutoff of $4 \AA$ was applied to all non bonded interactions, and $\mathrm{SHAKE}^{57}$ was employed to constraint all bonds involving hydrogen atoms. All simulations at this stage were performed using the sander module of AmberTools15. ${ }^{58}$

Set up of simulation systems. The $\beta$ LG dimer was constructed from the X-ray crystallographic structure 1BEB deposited in the Protein Data Bank, while our previously reported, ${ }^{22}$ docking-derived, model was used as the starting structure to build the $\beta$ LG-Hyp complex. Two disulfide bridges (Cys66-Cys160 and Cys106-Cys119) were set up on each monomer of $\beta$ LG. The simulated system was solvated on a truncated octahedron box of TIP3P water molecules and the appropriate number of $\mathrm{Na}^{+}$ counterions was added to neutralize the total charge. The ff14SBildn force field ${ }^{59}$ was used for the protein, while Hyp was parameterized using the general amber force field in conjunction with RESP (HF/6-31G(d)) charges, and Joung and Cheatham ${ }^{60,61}$ parameters to model the counterions, as implemented in the Antechamber module of AmberTools15 software package. Each system comprised around 60000 atoms, including the proteinligand complex, 900 DMSO molecules, 14000 water molecules and $9 \mathrm{Na}^{+}$counterions in a simulation box of $625000 \AA^{3}$.

The geometry of the system was minimized in five cycles that combined 3500 steps of steepest descent algorithm followed by 6500 of conjugate gradient. Thermalization was performed in 3 steps of $125 \mathrm{ps}$, where the temperature was gradually increased from $50 \mathrm{~K}$ to $298 \mathrm{~K}$, while the protein and ligands were restrained with a force constant that was concurrently reduced from $1 \mathrm{kcal} \mathrm{mol}^{-1} \AA^{-2}$ to $0.1 \mathrm{kcal} \mathrm{mol}^{-1} \AA^{-2}$. Prior to the production runs, each system was subjected to a $0.5 \mathrm{~ns}$ simulation on the NPT ensemble to equilibrate the density of the system. During the thermalization and equilibration stages a timestep of $1 \mathrm{fs}$ was employed using SHAKE to constraint bonds involving hydrogen atoms. The production runs consisted of $250 \mathrm{~ns}$ using SHAKE for bonds involving hydrogen atoms, a time step of $2 \mathrm{fs}$, periodic boundary conditions at constant volume and temperature $(298 \mathrm{~K}$; Langevin thermostat with a collision frequency of $3 \mathrm{ps}^{-1}$ ), particle mesh Ewald to handle long-range electrostatic interactions, and applying a cutoff of $10 \AA$ to all nonbonded interactions. The sander module was employed for the minimization stage, while the CUDA accelerated version of PMEMD ${ }^{62}$ was used thorough the heating, equilibration and production stages, with both modules being available in the standard distribution of AMBER15.

\section{Microbial strain growth and photoinactivation}

The bacterial strain used was Staphylococcus aureus CECT 239 obtained from the Spanish Type Culture Collection (CECT). Vegetative bacterial cells were grown in sterile Tryptic soy broth at $37^{\circ} \mathrm{C}$ until an optical density at $600 \mathrm{~nm}$ corresponding to 0.4 . The cells suspensions were then washed three times in sterile PBS by means of centrifugation and re-suspension, diluted 1:2 with a double concentrated solution containing the photosensitizer and incubated in the dark for $30 \mathrm{~min}$ at room temperature. For the photoinactivation experiments, the suspensions were placed in 96-wells plates, irradiated with green light for 
15 or $30 \mathrm{~min}$ (18 and $37 \mathrm{~J} \mathrm{~cm}^{-2}$ ) and serially diluted until $10^{-6}$ times the original concentration. Colony forming units (CFUs) were counted after $24 \mathrm{~h}$ incubation in the dark at $37{ }^{\circ} \mathrm{C}$ to calculate the survival fraction. Experiments were carried out in duplicate.

\section{Acknowledgements}

CV acknowledges S. E. Braslavsky for the kind donation of the Spectron Laser. This work has been supported by the Spanish MINECO, grant No. CTQ2013-48767-C3-1-R, and Generalitat de Catalunya, SGR2014-1189. FJL acknowledges the ICREA foundation for financial support.

\section{Notes and references}

1 T. Dai, Y.-Y. Huang and M. R. Hamblin, Photodiagn. Photodyn. Ther., 2009, 6, 170-188.

2 G. B. Kharkwal, S. K. Sharma, Y. Y. Huang, T. Dai and M. R. Hamblin, Lasers Surg. Med., 2011, 43, 755-767.

3 Z. Luksiene and L. Brovko, Food Eng. Rev., 2013, 5, 185-199.

4 Singlet Oxygen. Applications in Biosciences and Nanosciences, ed. S. Nonell and C. Flors, London, 2016.

5 G. Jori, M. Camerin, M. Soncin, L. Guidolin and O. Coppellotti, in Photodynamic Inactivation of Microbial Pathogens: Medical and Environmental Applications, ed. M. R. Hamblin and G. Jori, The Royal Society of Chemistry, 2011, pp. 1-18.

6 E. Alves, M. A. F. Faustino, M. G. P. M. S. Neves, A. Cunha, J. Tome and A. Almeida, Future Med. Chem., 2014, 6, 141-164.

7 N. Duràn and P. S. Song, Photochem. Photobiol., 1986, 43, 677-680.

8 K. Kairyte, S. Lapinskas, V. Gudelis and Z. Luksiene, J. Appl. Microbiol., 2012, 112, 1144-1151.

9 C. M. N. Yow, H. M. Tang, E. S. M. Chu and Z. Huang, Photochem. Photobiol., 2012, 88, 626-632.

10 N. Nafee, A. Youssef, H. El-Gowelli, H. Asem and S. Kandil, Int. J. Pharm., 2013, 454, 249-258.

11 J. M. Jacobson, L. Feinman, L. Liebes, N. Ostrow, V. Koslowski, A. Tobia, B. E. Cabana, D. H. Lee, J. Spritzler and A. M. Prince, Antimicrob. Agents Chemother., 2001, 45, 517-524.

12 D. S. English, K. Das, J. M. Zenner, W. Zhang, G. A. Kraus, R. C. Larock and J. W. Petrich, J. Phys. Chem. A, 1997, 101, 3235-3240.

13 A. Darmanyan, L. Burel, D. Eloy and P. Jardon, J. Chim. Phys., 1994, 91, 1774-1785.

14 M. Roslaniec, H. Weitman, D. Freeman, Y. Mazur and B. Ehrenberg, J. Photochem. Photobiol., B, 2000, 57, 149-158.

15 T. Yamazaki, N. Ohta, I. Yamazaki and P. S. Song, J. Phys. Chem., 1993, 97, 7870-7875.

16 J. L. Wynn and T. M. Coton, J. Phys. Chem., 1995, 99, 4317-4323.

17 M. Fadel, K. Kassab and T. Youssef, Lasers in Medical Science, 2010, 25, 675-683.

18 M. Zeisser-Labouèbe, N. Lange, R. Gurny and F. Delie, Int. J. Pharm., 2006, 326, 174-181.
19 O. Planas, E. Boix-Garriga, B. Rodriguez-Amigo, J. Torra, R. Bresolí-Obach, C. Flors, C. Viappiani, M. Agut, R. RuizGonzález and S. Nonell, in Photochemistry, ed. A. Albini and E. Fasani, The Royal Society of Chemistry, London, 2014.

20 J. Comas-Barceló, B. Rodríguez-Amigo, S. Abbruzzetti, P. D. Rey-Puech, M. Agut, S. Nonell and C. Viappiani, RSC Adv., 2013, 3, 17874-17879.

21 P. Delcanale, F. Pennacchietti, G. Maestrini, B. RodríguezAmigo, P. Bianchini, A. Diaspro, A. Iagatti, B. Patrizi, P. Foggi, M. Agut, S. Nonell, S. Abbruzzetti and C. Viappiani, Sci. Rep., 2015, 5, 15564.

22 B. Rodríguez-Amigo, P. Delcanale, G. Rotger, J. JuárezJiménez, S. Abbruzzetti, A. Summer, M. Agut, F. J. Luque, S. Nonell and C. Viappiani, J. Dairy Sci., 2015, 98, 89-94.

23 T. Arakawa, Y. Kita and S. N. Timasheff, Biophys. Chem., 2007, 131, 62-70.

24 D. Mercadante, L. D. Melton, G. E. Norris, T. S. Loo, M. A. K. Williams, R. C. J. Dobson and G. B. Jameson, Biophys. J., 2012, 103, 303-312.

25 E. Dufour, C. Bertrand-Harb and T. Haertlé, Biopolymers, 1993, 33, 589-598.

26 P. Miskovsky, D. Jancura, S. Sanchez-Cortez, E. Kocisova and L. Chinsky, J. Am. Chem. Soc., 1998, 120, 6374-6379.

27 D. Magde, E. Elson and W. W. Webb, Phys. Rev. Lett., 1972, 29, 705-708.

28 E. Haustein and P. Schwille, Annu. Rev. Biophys. Biomol. Struct., 2007, 36, 151-169.

29 A. Losi, Photochem. Photobiol., 1997, 65, 791-801.

30 C. Mattos and D. Ringe, Nat. Biotechnol., 1996, 14, 595-599.

31 E. Liepinsh and G. Otting, Nat. Biotechnol., 1997, 15, 264-268.

32 A. C. English, C. R. Groom and R. E. Hubbard, Protein Eng., 2001, 14, 47-59.

33 D. Alvarez-Garcia and X. Barril, J. Med. Chem., 2014, 57, 8530-8539.

34 H. Vondracek, J. Dielmann-Gessner, W. Lubitz, M. Knipp and M. Havenith, J. Chem. Phys., 2014, 141, 22 D534.

35 S. Ebbinghaus, S. J. Kim, M. Heyden, X. Yu, U. Heugen, M. Gruebele, D. M. Leitner and M. Havenith, Proc. Natl. Acad. Sci. U. S. A., 2007, 104, 20749-20752.

36 S. Ebbinghaus, K. Meister, B. Born, A. L. DeVries, M. Gruebele and M. Havenith, J. Am. Chem. Soc., 2010, 132, 12210-12211.

37 J. M. G. Cowie and P. M. Toporowski, Can. J. Chem., 1961, 39, 2240-2243.

38 R. G. LeBel and D. A. I. Goring, J. Chem. Eng. Data, 1962, 7, 100-101.

39 F. Gai, M. J. Fehr and J. W. Petrich, J. Phys. Chem., 1994, 98, 5784-5795.

40 F. Gai, M. J. Fehr and J. W. Petrich, J. Phys. Chem., 1994, 98, 8352-8358.

41 K. Das, A. V. Smirnov, M. D. Snyder and J. W. Petrich, J. Phys. Chem. B, 1998, 102, 6098-6106.

42 E. I. Kapinus, Biophysics, 2010, 55, 188-193.

43 H. Lawrence Clever, R. Battino, H. Miyamoto, Y. Yampolski and C. L. Young, J. Phys. Chem. Ref. Data, 2014, 43, 033102. 
44 C. Cecchini, A. Cresci, M. M. Coman, M. Ricciutelli, G. Sagratini, S. Vittori, D. Lucarini and F. Maggi, Planta Med., 2007, 73, 564-566.

45 K. Aponiene, E. Paskeviciute, I. Reklaitis and Z. Luksiene, J. Food Eng., 2015, 144, 29-35.

46 S. Nonell and S. E. Braslavsky, in Singlet oxygen, $U V-A$, and Ozone, ed. L. P. A. H. Sies, Academic Press, San Diego, 2000, pp. 37-49.

47 S. Abbruzzetti, S. Bruno, S. Faggiano, E. Grandi, A. Mozzarelli and C. Viappiani, Photochem. Photobiol. Sci., 2006, 5, 1109-1120.

48 D. Alvarez-Garcia and X. Barril, J. Chem. Theory Comput., 2014, 10, 2608-2614.

49 C. C. G. Inc., ed. S. 1010 Sherbooke St. West, Montreal, QC, Canada, H3A 2R7, 2016.

50 M. J. Frisch, G. W. Trucks, H. B. Schlegel, G. E. Scuseria, M. A. Robb, J. R. Cheeseman, G. Scalmani, V. Barone, B. Mennucci, G. A. Petersson, H. Nakatsuji, M. Caricato, X. Li, H. P. Hratchian, A. F. Izmaylov, J. Bloino, G. Z. J. L. Sonnenberg, M. Hada, M. Ehara, K. T. R. Fukuda, J. Hasegawa, M. Ishida, T. N. Y. Honda, O. Kitao, H. Nakai, T. Vreven, J. J. A. Montgomery, J. E. Peralta, F. Ogliaro, M. Bearpark, J. J. Heyd, E. Brothers, K. N. Kudin, V. N. Staroverov, R. Kobayashi, J. Normand, K. Raghavachari, A. Rendell, J. C. Burant, S. S. Iyengar, J. Tomasi, M. Cossi, N. Rega, J. M. Millam, M. Klene, J. E. Knox, J. B. Cross, V. B. C. Adamo, J. Jaramillo, R. Gomperts, R. E. Stratmann, O. Y. A. J. Austin, R. Cammi, C. Pomelli, J. W. Ochterski, R. L. Martin, K. Morokuma, V. G. Z. G. A. Voth, P. Salvador, J. J. Dannenberg, S. Dapprich, A. D. Daniels, Ö. Farkas, J. B. Foresman, J. V. Ortiz, J. Cioslowski and D. J. Fox, Gaussian 09, Revision C.01, Gaussian, Inc., Wallingford CT, 2009.
51 T. Fox and P. A. Kollman, J. Phys. Chem. B, 1998, 102, 8070-8079.

52 C. I. Bayly, P. Cieplak, W. Cornell and P. A. Kollman, J. Phys. Chem., 1993, 97, 10269-10280.

53 J. Wang, R. M. Wolf, J. W. Caldwell, P. A. Kollman and D. A. Case, J. Comput. Chem., 2004, 25, 1157-1174.

54 J. Wang, R. M. Wolf, J. W. Caldwell, P. A. Kollman and D. A. Case, J. Comput. Chem., 2005, 26, 114.

55 W. L. Jorgensen, J. Chandrasekhar, J. D. Madura, R. W. Impey and M. L. Klein, J. Chem. Phys., 1983, 79, 926-935.

56 D. J. Price and C. L. Brooks, J. Chem. Phys., 2004, 121, 10096-10103.

57 S. Miyamoto and P. A. Kollman, J. Comput. Chem., 1992, 13, 952-962.

58 D. A. Case, T. A. Darden, I. T. E. Cheatham, C. L. Simmerling, J. Wang, R. E. Duke, R. Luo, R. C. Walker, W. Zhang, K. M. Merz, B. Roberts, S. Hayik, A. Roitberg, G. Seabra, J. Swails, A. W. Götz, I. Kolossváry, K. F. Wong, F. P. J. Vanicek, R. M. Wolf, J. Liu, X. Wu, S. R. B. T. Steinbrecher, H. Gohlke, Q. Cai, X. Ye, J. Wang, M.-J. Hsieh, G. Cui, D. R. Roe, D. H. Mathews, M. G. Seetin, R. Salomon-Ferrer, C. Sagui, V. Babin, T. Luchko, S. Gusarov and A. K. P. A. Kollman, AMBER12, San Francisco, 2012.

59 J. A. Maier, C. Martinez, K. Kasavajhala, L. Wickstrom, K. E. Hauser and C. Simmerling, J. Chem. Theory Comput., 2015, 11, 3696-3713.

60 I. S. Joung and T. E. Cheatham, J. Phys. Chem. B, 2008, 112, 9020-9041.

61 I. S. Joung and T. E. Cheatham, J. Phys. Chem. B, 2009, 113, 13279-13290.

62 R. Salomon-Ferrer, A. W. Goetz, D. Poole, S. Le Grand and R. C. Walker, J. Chem. Theory Comput., 2013, 9, 3878-3888. 